Ferrata Storti Foundation

\title{
Clinical and molecular characteristics of MEF2D fusion-positive B-cell precursor acute lymphoblastic leukemia in childhood, including a novel translocation resulting in MEF2D-HNRNPH1 gene fusion
}

Haematologica 2019

Volume 104(1):128-137

\section{Correspondence:}

oki-kn@ncchd.go.jp

Received: December 11, 2017.

Accepted: August 29, 2018.

Pre-published: August 31, 2018.

doi:10.3324/haematol.2017.186320

Check the online version for the most updated information on this article, online supplements, and information on authorship \& disclosures: www.haematologica.org/content/104/1/128

(C)2019 Ferrata Storti Foundation

Material published in Haematologica is covered by copyright. All rights are reserved to the Ferrata Storti Foundation. Use of published material is allowed under the following terms and conditions:

https://creativecommons.org/licenses/by-nc/4.0/legalcode. Copies of published material are allowed for personal or internal use. Sharing published material for non-commercial purposes is subject to the following conditions:

https://creativecommons.org/licenses/by-nc/4.0/leǵalcode, sect. 3. Reproducing and sharing published material for commercial purposes is not allowed without permission in writing from the publisher.
Kentaro Ohki, ${ }^{1}$ Nobutaka Kiyokawa, ${ }^{1}$ Yuya Saito, ${ }^{1,2}$ Shinsuke Hirabayashi, ${ }^{1,3}$ Kazuhiko Nakabayashi, ${ }^{4}$ Hitoshi Ichikawa, ${ }^{5}$ Yukihide Momozawa, ${ }^{6}$ Kohji Okamura, ${ }^{7}$ Ai Yoshimi, ${ }^{1,8}$ Hiroko Ogata-Kawata, ${ }^{4}$ Hiromi Sakamoto, ${ }^{5}$ Motohiro Kato, ${ }^{1}$ Keitaro Fukushima, ${ }^{9}$ Daisuke Hasegawa, ${ }^{3}$ Hiroko Fukushima, ${ }^{10}$ Masako Imai, ${ }^{11}$ Ryosuke Kajiwara, ${ }^{12}$ Takashi Koike, ${ }^{13}$ Isao Komori, ${ }^{14}$ Atsushi Matsui, ${ }^{15}$ Makiko Mori, ${ }^{16}$ Koichi Moriwaki, ${ }^{17}$ Yasushi Noguchi, ${ }^{18}$ Myoung-ja Park, ${ }^{19}$ Takahiro Ueda, ${ }^{20}$ Shohei Yamamoto, ${ }^{21}$ Koichi Matsuda, ${ }^{22}$ Teruhiko Yoshida, ${ }^{5}$ Kenji Matsumoto, ${ }^{23}$ Kenichiro Hata, ${ }^{4}$ Michiaki Kubo, ${ }^{6}$ Yoichi Matsubara, ${ }^{24}$ Hiroyuki Takahashi, ${ }^{25}$ Takashi Fukushima, ${ }^{26}$ Yasuhide Hayashi, ${ }^{27}$ Katsuyoshi Koh, ${ }^{16}$ Atsushi Manabe ${ }^{3}$ and Akira Ohara ${ }^{25}$ for the Tokyo Children's Cancer Study Group (TCCSG)

${ }^{1}$ Department of Pediatric Hematology and Oncology Research, National Research Institute for Child Health and Development, Setagaya-ku, Tokyo; ${ }^{2}$ Department of Hematology/Oncology, Tokyo Metropolitan Children's Medical Center, Fuchu-shi; ${ }^{3}$ Department of Pediatrics, St. Luke's International Hospital, Chuo-ku, Tokyo; ${ }^{4}$ Department of Maternal-Fetal Biology, National Research Institute for Child Health and Development, Setagaya-ku, Tokyo; ${ }^{5}$ Fundamental Innovative Oncology Core, National Cancer Center Research Institute, Chuo-ku, Tokyo; ‘'Laboratory for Genotyping Development, RIKEN Center for Integrative Medical Sciences (IMS), Yokohama-shi, Kanagawa; ${ }^{7}$ Department of Systems BioMedicine, National Research Institute for Child Health and Development, Setagaya-ku, Tokyo; ${ }^{8}$ Division of Pediatric Hematology and Oncology, Ibaraki Children's Hospital, Mito-shi; ' Department of Pediatrics, Dokkyo Medical University, Tochigi; ${ }^{10}$ Department of Pediatrics, University of Tsukuba Hospital, Ibaraki; ${ }^{11}$ Department of Pediatrics, Japanese Red Cross Musashino Hospital, Tokyo; ${ }^{12}$ Department of Pediatrics, Yokohama City University Hospital, Kanagawa; ${ }^{13}$ Department of Pediatrics, Tokai University School of Medicine, Kanagawa; ${ }^{14}$ Department of Pediatrics, Matsudo City Hospital, Chiba; ${ }^{15}$ Department of Pediatrics, Japanese Red Cross Maebashi Hospital, Gunma; ${ }^{16}$ Department of Hematology/Oncology, Saitama Children's Medical Center; ${ }^{17}$ Department of Pediatrics, Saitama Medical Center, Saitama Medical University; ${ }^{18}$ Department of Pediatrics, Japanese Red Cross Narita Hospital, Chiba; ${ }^{19}$ Department of Hematology/Oncology, Gunma Children's Medical Center, Shibukawa-shi; ${ }^{20}$ Department of Pediatrics, Nippon Medical School, Bunkyo-ku, Tokyo; ${ }^{21}$ Department of Pediatrics, Showa University Fujigaoka Hospital, Yokohama-shi, Kanagawa; ${ }^{22}$ Laboratory of Clinical Genome Sequencing Department of Computational Biology and Medical Sciences Graduate School of Frontier Sciences, The University of Tokyo, Minato-ku; ${ }^{23}$ Department of Allergy and Clinical Immunology, National Research Institute for Child Health and Development, Setagaya-ku, Tokyo; ${ }^{24}$ Director, National Research Institute for Child Health and Development, Setagaya-ku, Tokyo; ${ }^{25}$ Department of Pediatrics, Toho University Omori Medical Center, Tokyo; ${ }^{26}$ Department of Child Health, Faculty of Medicine, University of Tsukuba, Ibaraki and ${ }^{27}$ Institute of Physiology and Medicine, Jobu University, Takasaki-shi, Gunma, Japan

\section{ABSTRACT}

Tusion genes involving $M E F 2 D$ have recently been identified in pre- cursor B-cell acute lymphoblastic leukemia, mutually exclusive of 1 the common risk stratifying genetic abnormalities, although their true incidence and associated clinical characteristics remain unknown. We identified 16 cases of acute lymphoblastic leukemia and 1 of lymphoma harboring MEF2D fusions, including MEF2D-BCL9 ( $\mathrm{n}=10$ ), MEF2D-HNRNPUL1 ( $\mathrm{n}=6$ ), and one novel MEF2D-HNRNPH1 fusion. The incidence of $M E F 2 D$ fusions overall was $2.4 \%$ among consecutive precursor B-cell acute lymphoblastic leukemia patients enrolled onto a single clinical trial. They frequently showed a cytoplasmic $\mu$ chain-positive pre-B immunophenotype, and often expressed an aberrant CD5 antigen. Besides up- and down-regulation of HDAC9 and MEF2C, elevated GATA3 expression was also a characteristic feature of MEF2D 
fusion-positive patients. Mutations of PHF6, recurrent in T-cell acute lymphoblastic leukemia, also showed an unexpectedly high frequency $(50 \%)$ in these patients. MEF2D fusion-positive patients were older (median age 9 years) with elevated WBC counts (median: 27,300/ $\mu \mathrm{l}$ ) at presentation and, as a result, were mostly classified as NCI high risk. Although they responded well to steroid treatment, MEF2D fusion-positive patients showed a significantly worse outcome, with $53.3 \%$ relapse and subsequent death. Stem cell transplantation was ineffective as salvage therapy. Interestingly, relapse was frequently associated with the presence of CDKN2A/CDKN2B gene deletions. Our observations indicate that MEF2D fusions comprise a distinct subgroup of precursor B-cell acute lymphoblastic leukemia with a characteristic immunophenotype and gene expression signature, associated with distinct clinical features.

\section{Introduction}

Precursor B-cell acute lymphoblastic leukemia (B-ALL) is a heterogeneous disease characterized by a variety of genetic abnormalities. ${ }^{1}$ In approximately one-quarter of B-ALL patients, known as the B-other-subgroup, the known major risk-stratifying cytogenetic abnormalities are absent. ${ }^{2}$ However, recent studies using advanced analytical approaches have described a range of novel genetic subgroups among B-other-ALL. ${ }^{3-11}$

The myocyte enhancer factor 2D (MEF2D) gene, located at $1 \mathrm{q} 22$, is present among these newly identified rearrangements in B-other-ALL. ${ }^{6,710,12-14}$ Seven known fusion partners: $B$-cell CLL/lymphoma 9 (BCL9, 1q21), heterogeneous nuclear ribonucleoprotein U-like 1 (HNRNPUL1, 19q13.2), deleted in azoospermia-associated protein 1 (DAZAP1, 19p13.3), colony stimulating factor 1 receptor (CSF1R, 5q32), synovial sarcoma translocation, chromosome $18(S S 18,18 \mathrm{q} 11.2)$, signal transducer and activator of transcription 6 (STAT6, 12q13.3), and Forkhead Box J2 (FOXJ2, 12p13.31) have been described, mostly among childhood and young adult B-ALL. The MEF2D gene encodes a member of the transcription factor family involved in the control of muscle and neuronal cell differentiation and development, which is regulated by class II histone deacetylases. ${ }^{15-17}$ It has been reported that rearrangements result in enhanced MEF2D transcriptional activity and lymphoid transformation, thus contributing to the development of a distinct subtype of high-risk leukemia.,10 However, the true incidence and clinical characteristics, including outcome, of patients with B-ALL harboring MEF2D fusion genes remains unknown.

In this study, we report the detailed analysis of a subgroup of B-ALL with MEF2D fusions within a Japanese pediatric ALL cohort. The heterogeneous nuclear ribonucleoprotein H1 gene (HNRNPH1), encoding another family member of heterogeneous nuclear ribonucleoprotein, ${ }^{18}$ was identified as a new fusion partner of the MEF2D gene. Novel immunophenotypic characteristics and accompanying genetic abnormalities as well as distinctive clinical features of B-ALL harboring MEF2D fusions are evaluated and discussed.

\section{Methods}

\section{Patient selection and sample preparation}

RNA and DNA samples, obtained from pediatric B-ALL patients and stored in the Tokyo Children's Cancer Study Group (TCCSG) biobank ${ }^{5,11}$ were used in this study (Online Supplementary Figure S1, Table S1). As indicated in Online Supplementary Table S1, the majority of cases originated from the
TCCSG L04-16 study ${ }^{19}$ while others, including 2 B-lymphoblastic lymphoma (LBL) patients, originated from different cohorts. All investigations were approved by the institutional review boards and informed consent or assent was obtained from parents or guardians based on their age and level of understanding, as described previously. ${ }^{5,11}$ Online Supplementary Figure S1 shows the analysis carried out on each case.

Total RNA and genomic DNA were extracted from bone marrow or peripheral blood of patients using the miRNeasy Mini Kit and the OIAamp DNA Mini Kit (Qiagen, Inc., Valencia, CA, USA), respectively.

In this paper, B-other-ALL is defined as B-ALL lacking the major risk stratifying genetic abnormalities, including high hyperdiploidy ( $\geq 51$ chromosomes or DNA index $\geq 1.16$ ), low hypodiploidy/near haploidy ( $\leq 44$ chromosomes), fusions of ETV6-RUNX1, TCF3-PBX1, TCF3-HLF, BCR-ABL1, and MLL rearrangements as well as more recently identified genetic abnormalities, including rearrangements of CRLF2 and ZNF384, Ph-like ALL-related tyrosine kinase fusions as well as $M E F 2 D$ fusions (Online Supplementary Table S1).

\section{Whole transcriptome sequencing and RT-PCR}

From previous whole transcriptome sequencing (WTS) of Bother-ALL, we identified cases with ZNF384 fusions ${ }^{11}$ as well as other abnormalities (Online Supplementary Figure S1, Table S1). ${ }^{19}$ ${ }^{21}$ We re-analyzed remaining 153 WTS data manipulated by "deFuse", ${ }^{22}$ an algorithm for gene fusion discovery, and investigated the presence of MEF2D fusions. Details of this data analysis have been described previously. ${ }^{11}$ RT-PCR followed by Sanger sequencing was performed to confirm and screen for fusion transcripts, as described previously, ${ }^{5,11}$ using the primers listed in Online Supplementary Table S2.

\section{Multiplex Ligation-dependent Probe Amplification (MLPA)}

MLPA analyses were performed on genomic DNA using two types of SALSA Reference Kits, P335 and P383 (MRC Holland, Amsterdam, the Netherlands), according to the manufacturer's instructions. After separation of amplified products, using the ABI3130 Genetic Analyzer (Applied Biosystems, Foster City, CA, USA), the results were analyzed using Gene Mapper Software (Applied Biosystems) and data, including informative headers, electropherograms, ratio plots, validation boxes, and report tables were obtained. In this study, we present only results of deletions of the exons targeted in these kits.

\section{Whole exome sequencing (WES)}

Exome libraries prepared from 100 ng of genomic DNA were sequenced using SBS v.4 reagents with the HiSeq2500 sequencing system. Details of whole exome data analyses have been described previously. ${ }^{11}$ 


\section{Microarray and data analyses}

The gene expression signature for MEF2D fusion-positive BALL was investigated by DNA microarray-based expression profiling using Human Genome U133 Plus 2.0 Arrays (Affymetrix, Santa Clara, CA, USA). Data were normalized and filtered as described previously. ${ }^{11}$ Further details are provided in Online Supplementary Methods.

\section{Statistical analysis}

Mutual univariate analyses of characteristics were conducted using Fisher's exact test or the $\chi^{2}$ test for qualitative variables. Overall survival (OS) and event-free survival (EFS) were estimated by the Kaplan-Meier method and compared by the log-rank test. Analyses were performed using Prism software, version 6.0 (GraphPad Software, Inc., La Jolla, CA, USA).

Table 1. Characteristics of MEF2D fusion-positive cases.

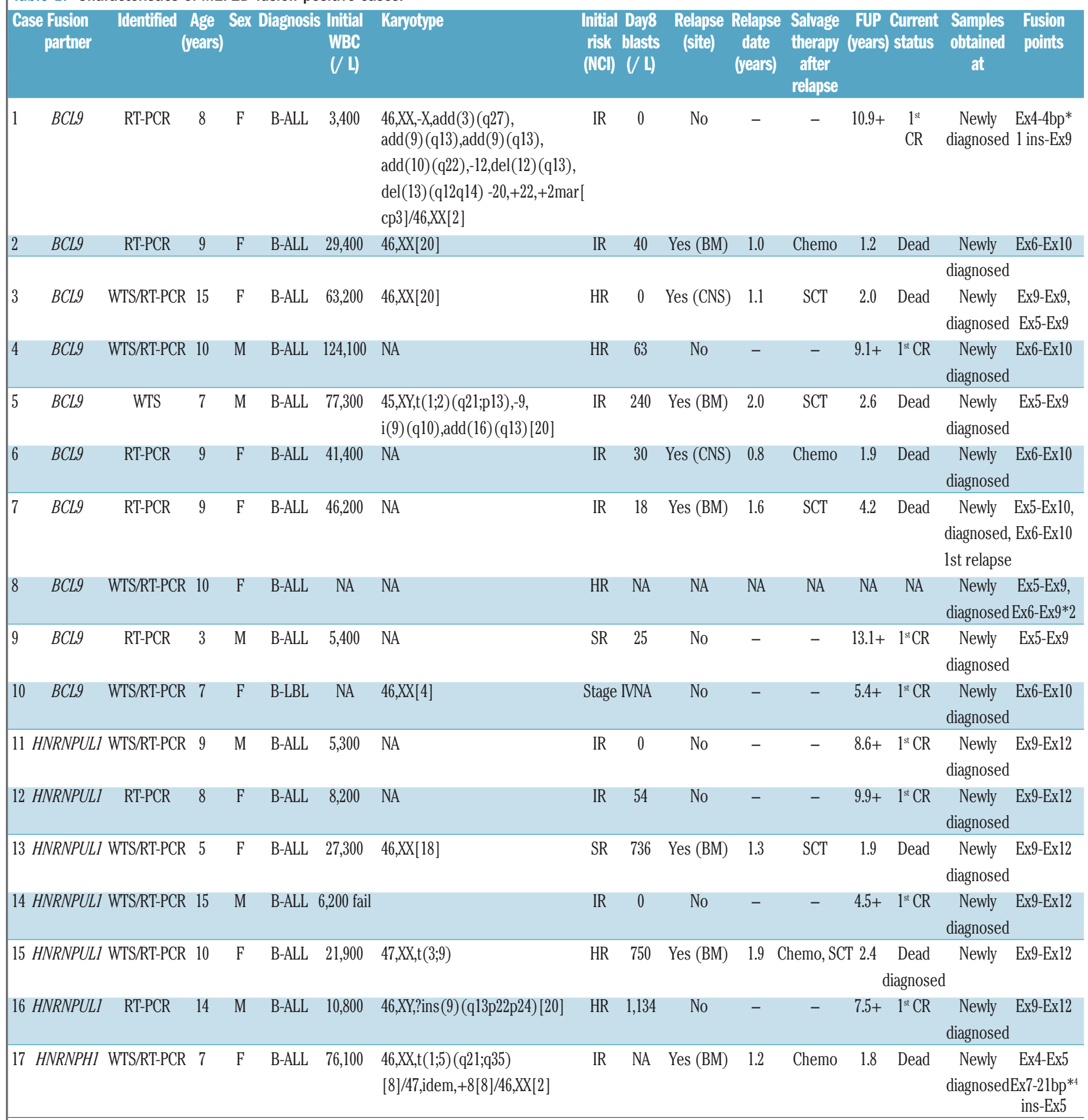




\section{Results}

Detection of MEF2D fusions in pediatric B-ALL patients

Among the 328 selected RNA samples from B-ALL patients (Online Supplementary Figure S1) analyzed by WTS and/or RT-PCR followed by Sanger sequencing, we iden- tified 9 and 6 patients with MEF2D-BCL9 and MEF2DHNRNPUL1, respectively (Table 1, Online Supplementary Table S1 and S3, Figure 1 and Online Supplementary Figure S1). Of note, one case of each abnormality was identified by gene expression profiling (details in Online Supplementary Information). We also identified an additional case with MEF2D-BCL9 (Table 1, Case 10) in B-LBL. As

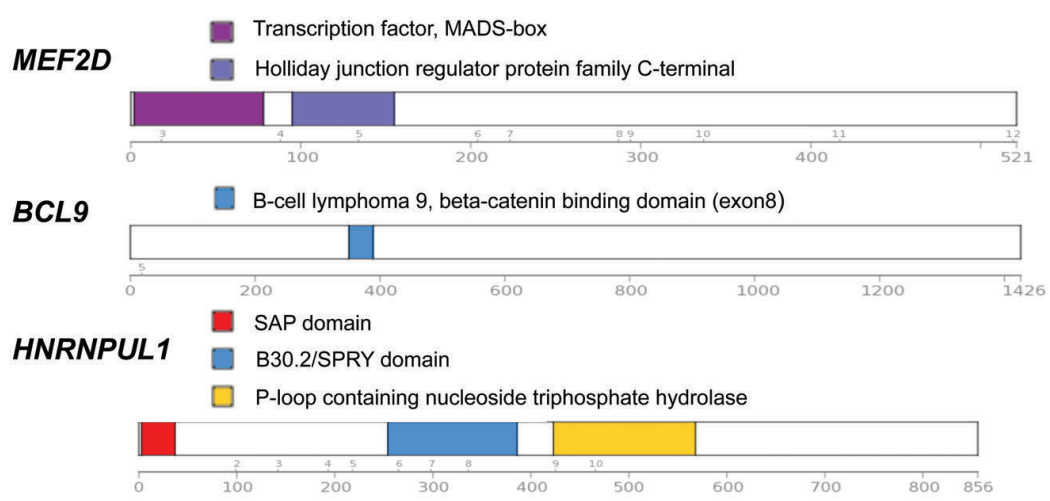

HNRNPH1 RNA recognition motif domain

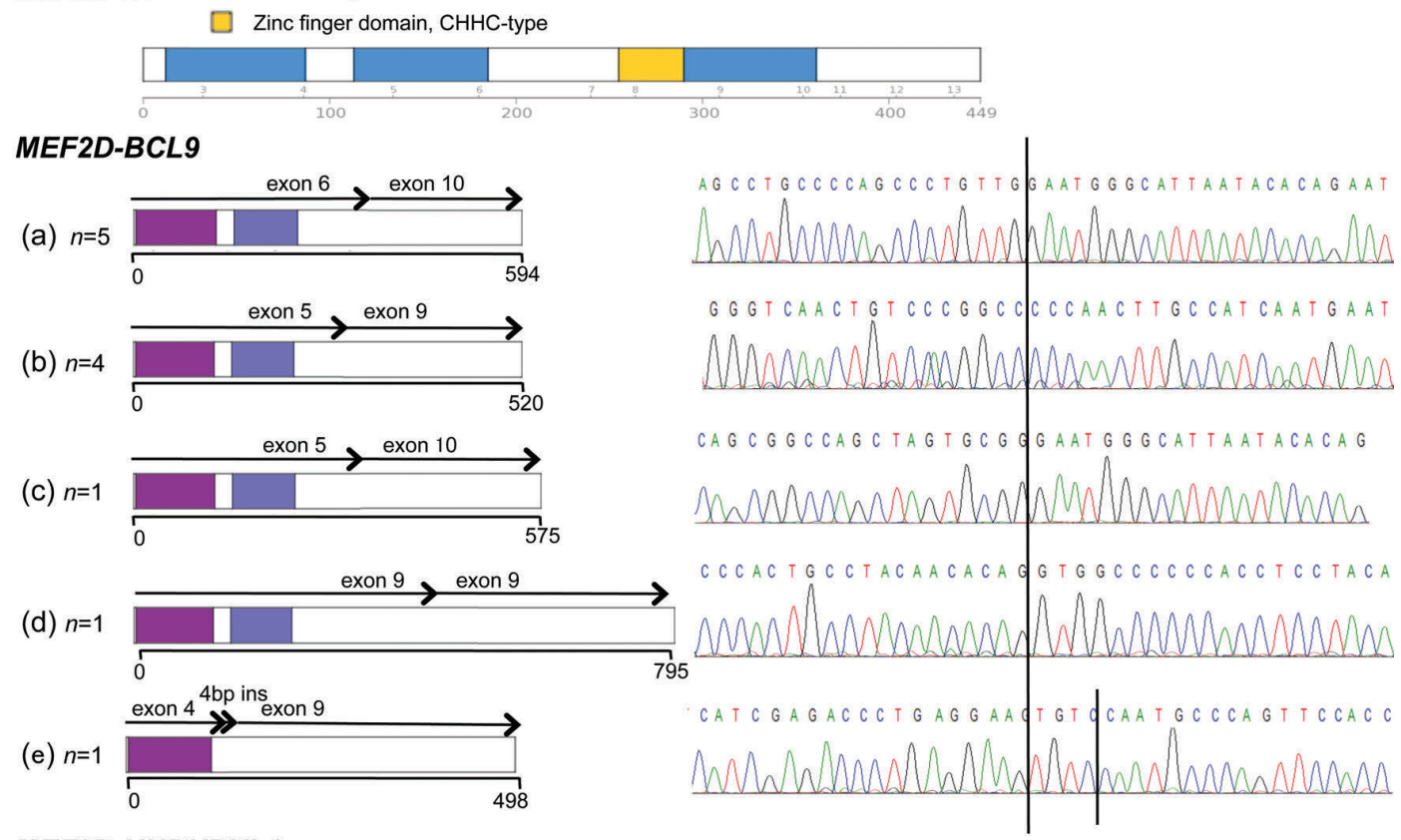

MEF2D-HNRNPUL1
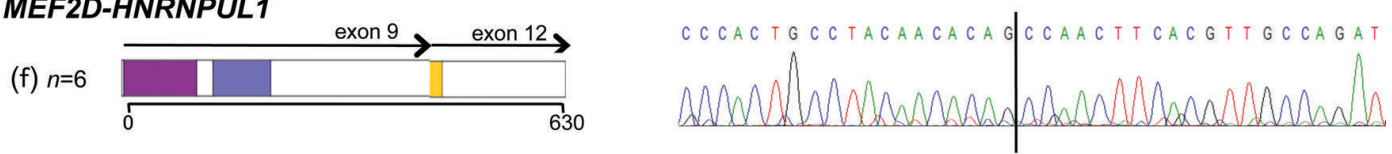

MEF2D-HNRNPH1

(g) $n=1$
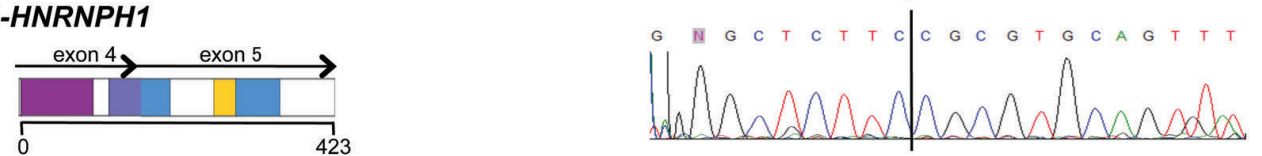

(h) $n=1$
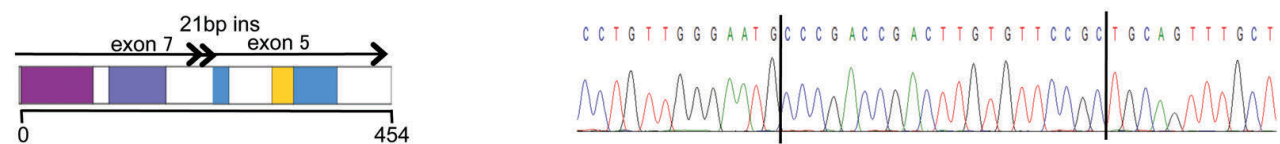

Figure 1. Structures of the MEF2D fusions. Structures of fusion proteins and nucleotide sequences of (a) - (e) MEF2D-BCL9, (f) MEF2D-HNRNPUL1, (g) and (h) MEF2D-HNRNPH1. The red arrowhead shows the donor and acceptor site breakpoint. The number of patients in whom a particular fusion isoform was found is indicated on the right. 
well as the known MEF2D fusions, we identified MEF2DHNRNPH1 as a novel fusion in 1 patient (Figure 1, Table 1, Case 17). Among the L04-16/L06-16 cohort, ${ }^{11,19}$ comprising a consecutive series of 290 B-ALL patients, including 126 classified as B-other-ALL, 5 MEF2D-BCL9 and 2 MEF2DHNRNPUL1 patients were identified (Online Supplementary Table S1 and Figure S1). The incidence of $M E F 2 D$ fusions in childhood ALL, calculated from this cohort, was $5.6 \%$ in B-other-ALL and $2.4 \%$ in B-ALL overall. MEF $2 D-B C L 9$ was the most recurrent, at a frequency of $4.0 \%$ in B-other-ALL and $1.7 \%$ in B-ALL overall.

\section{Structure of MEF2D fusions}

The structure and sequences of $M E F 2 D-B C L 9$ as well as a schematic representation of the predicted fusion proteins, are depicted in Figure 1, Table 1 and Online
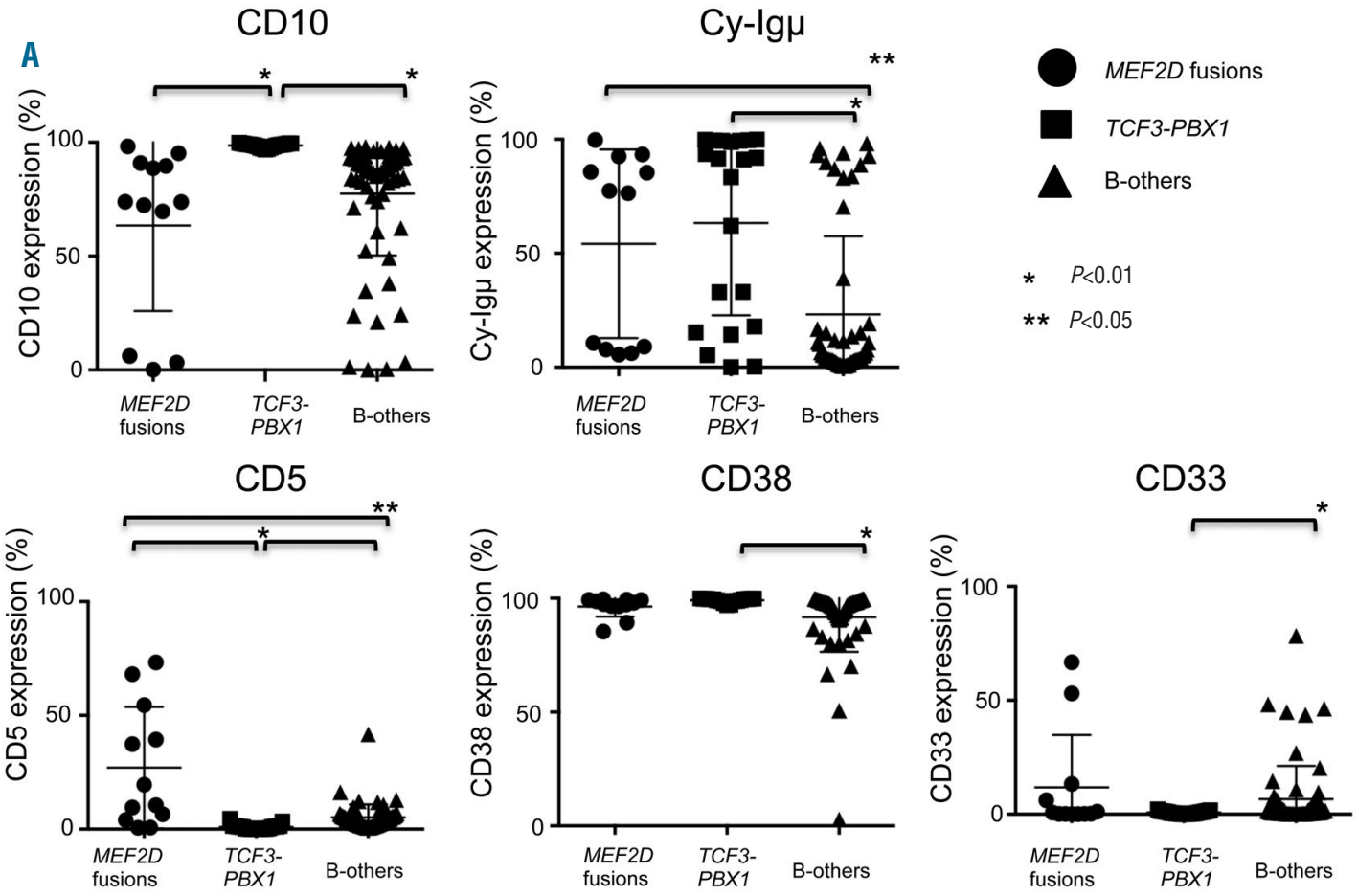

B

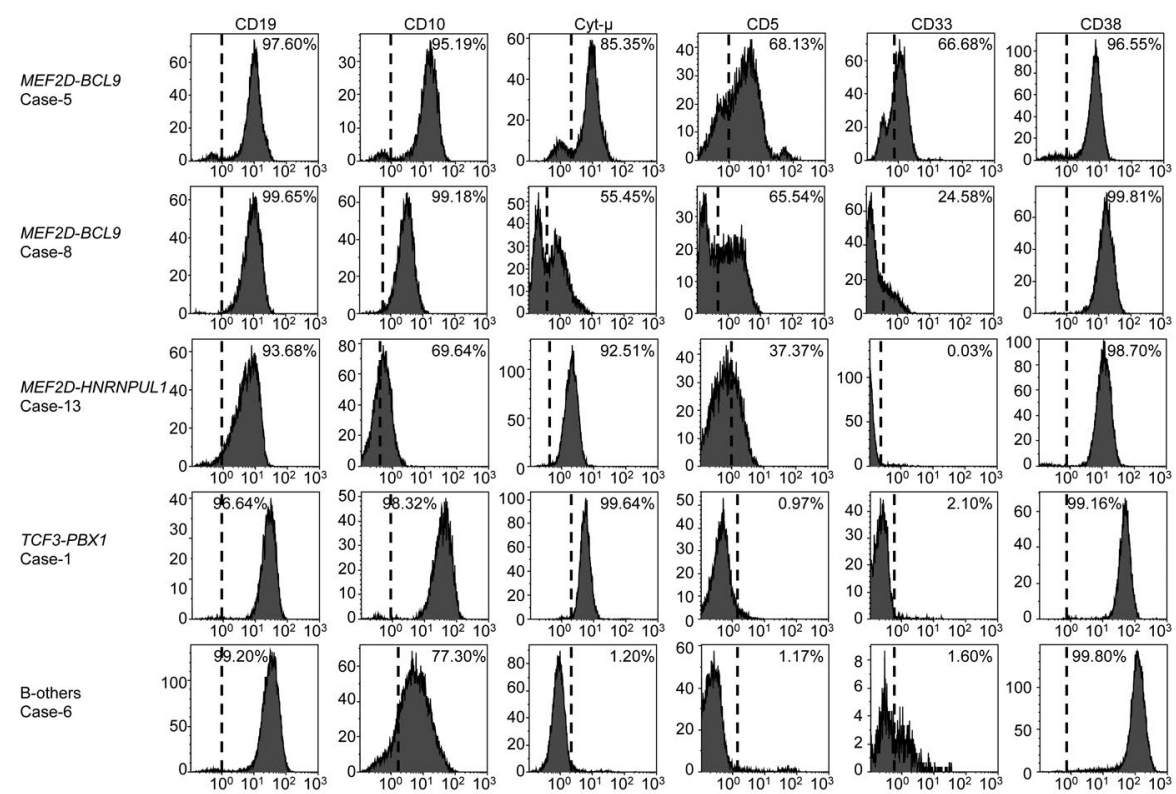

Figure 2. Immunophenotypic characteristics of B-ALL patients with MEF2D fusions. (A) The positivity (percentage) of CD10, cytoplasmic $\mu$, CD5, CD38, and CD33 of MEF2D fusion-positive, TCF3-PBX1-positive, and B-other patients are plotted as a scattergram. A detailed list of positivity for each immunophenotypic marker of the patients is presented in Online Supplementary Table S4. (B) Typical histograms of CD10, CD19, cytoplasmic $\mu$, aberrant CD5, CD33, and CD38 of MEF2D-BCL9, MEF2D-HNRNPUL1, TCF3-PBX1, and B-other patients are indicated with a positive rate (\%). X-axis, fluorescence intensity; Y-axis, relative cell number. 
Supplementary Table S3. Five isoforms of MEF2D-BCL9 fusion were identified in 10 patients, including one case in which different isoforms were present together. Most frequently, exon 6 of $M E F 2 D$ was fused in-frame to exon 10 of BCL9 in 5 patients (Figure 2A, Type (a)), in accordance with previous reports. 10 Exons 5 and 6 of MEF2D were also fused to exon 9 of BCL9 in 4 and 1 patient, respectively (Types (b) and (c)). In addition, we identified two novel breakpoints (Types (d) and (e)). The predicted protein from all fusions retains the DNA-binding MADS domain of the MEF2D protein, while it lacks most of the c-terminal portion of MEF2D as well as most of the functional domains of BCL9.

The structure and sequences of MEF2D-HNRNPUL1 are also presented in Figure 1. Distinct from previous reports, only one isoform joining exon 9 of MEF2D to exon 12 of HNPNPUL1 (Type (f)) was observed among our 6 patients. In the case of MEF2D-HNRNPH1, two isoforms, in-frame fusions joining exons 4 and 7 of $M E F 2 D$ to exon 5 of HNPNPH1 (Type (g) and (h)), respectively, were identified in the same patient.

\section{Immunophenotypic characteristics of B-ALL patients with MEF2D fusions}

It has been reported that B-ALL patients with MEF2D fusions show dull or negative expression of CD10 and overexpression of CD38 antigens, based on immunophenotypic examination. ${ }^{10}$ Among our MEF2D fusion-positive cases, CD10 expression was lower than that seen in TCF3-PBX1-positive patients, but not B-others-ALL, as shown in Figure 2, Online Supplementary Figure S3 and Table S4. On the other hand, CD38 expression in MEF2D fusion-positive cases was higher than B-other-ALL patients, but not TCF3-PBX1-positive patients. We also observed that expression of the cytoplasmic $\mu$ chain was higher in MEF2D fusion-positive cases than that in B-
other-ALL patients, with more than $10 \%$ of blasts positive for cytoplasmic $\mu$ in $8 / 12$ patients (ranging from 5.50 to $99.74 \%$, mean: $54.14 \pm 41.38 \%$ ). Moreover, B-ALL patients with MEF2D fusions frequently exhibited aberrant CD5 expression, with more than $10 \%$ of the blasts positive for CD5 in $7 / 12$ patients (ranging from 0.60 to $73.30 \%$, mean: $27.01 \pm 26.67 \%$ ).

\section{Additional genetic abnormalities in MEF2D fusion-positive patients}

To elucidate the frequency of additional genetic abnormalities in B-ALL with MEF2D fusions, we initially performed MLPA on 16 DNA samples from patients with MEF2D fusions. As shown in Online Supplementary Table S5, MLPA analysis revealed that deleted or amplified regions of $I K Z F 1, C R L F 2, E B F 1, B T G 1, P H F 6, N F 1, E Z H 2$, $S U Z 12$, or PTEN were absent from MEF2D fusion-positive cases, although 11/16 exhibited CDKN2A/CDKN2B deletions at a frequency significantly higher than that among the B-other-ALL patients enrolled on L04-16/L0616 study $(P<0.001$, data not shown). Heterozygous deletions of LEF1, PAX5, and ETV6 were detected in 1 case each.

To further investigate the presence of additional genetic abnormalities affecting coding sequences in B-ALL with MEF2D fusions, we carried out WES on 3 DNA samples. We identified 16 mutations within genes that had been previously recognized within cancer: $A L K, A R F G E R 3$, BRCA1, BRMS1, C8orf4, ITIH1, MAPK13, NCOR2, NLE, NOTCH1, PHF3, PHF6, PHF10, PIK3R5, RB1, and TET1 (Online Supplementary Table S6).

As mutations involving NOTCH1 and PHF6 are known to be recurrent in T-ALL ${ }^{23-26}$ and NLE encodes the modulator of NOTCH1, ${ }^{27}$ we were encouraged to investigate abnormalities in NOTCH1 signaling pathway genes and other genes reported as targets of recurrent genetic abnormalities in T-ALL. Thus, we performed RT-PCR and

Table 2. Additional genetic abnormalities of MEF2D fusion-positive cases.

\begin{tabular}{|c|c|c|c|c|c|c|c|c|}
\hline Case & Fusion partner & $\begin{array}{c}\text { Gene } \\
\text { Chromosome }\end{array}$ & $\begin{array}{l}F B X W 7 \\
4 q 31.3\end{array}$ & $\begin{array}{c}\text { NLE } \\
17 q 12\end{array}$ & $\begin{array}{c}\text { NOTCH1 } \\
9 q 34.3\end{array}$ & $\begin{array}{c}\text { PHF6 } \\
\times(q 26.2\end{array}$ & $\begin{array}{c}\text { PTEN } \\
\text { 10q23.31 }\end{array}$ & $\begin{array}{c}W T 1 \\
11 p 13\end{array}$ \\
\hline 1 & BCL9 & & WT & WT & 6764 T C, M2255T & WT & WT & WT \\
\hline 2 & BCL9 & & WT & WT & WT & WT & WT & WT \\
\hline 3 & BCL9 & & 1282 C G, Q428E & WT & WT & $\mathrm{R} 15 \mathrm{fs}$ & WT & WT \\
\hline 4 & BCL9 & & WT & WT & $6920 \mathrm{~A} \mathrm{G}, \mathrm{Q} 2307 \mathrm{R}$ & $\mathrm{S} 66 \mathrm{C}$ & WT & WT \\
\hline 5 & BCL9 & & WT & WT & WT & WT & WT & WT \\
\hline 6 & BCL9 & & WT & WT & WT & K16I & $2 \mathrm{C} \rightarrow \mathrm{T}, \mathrm{P} 231 \mathrm{~L}$ & WT \\
\hline 7 & BCL9 & & WT & WT & WT & WT & WT & $1192 \mathrm{~T} \rightarrow \mathrm{A}, \mathrm{C} 398 \mathrm{~S}$ \\
\hline 8 & BCL9 & & WT & WT & WT & WT & WT & WT \\
\hline 9 & BCL9 & & WT & WT & WT & D309H & WT & WT \\
\hline 11 & HNRNPUL1 & & WT & WT & WT & X2 del (no start codon), C82Y & WT & WT \\
\hline 12 & HNRNPUL1 & & WT & WT & \multirow{2}{*}{ WT } & ex2 del (no start codon) & WT & WT \\
\hline 13 & HNRNPULI & & WT & WT & & WT & WT & WT \\
\hline 14 & HNRNPUL1 & & WT & WT & WT & WT & WT & WT \\
\hline 15 & HNRNPUL1 & & WT & WT & WT & WT & WT & WT \\
\hline 16 & HNRNPUL1 & & WT & WT & WT & D309H & WT & WT \\
\hline 17 & HNRNPHI & & WT & 904 C G, R302G & WT & $955 \mathrm{C} \rightarrow \mathrm{T}, \mathrm{R} 319 \mathrm{X}$ & WT & WT \\
\hline & & Frequency & $1 / 16$ & $1 / 16$ & $2 / 16$ & $8 / 16$ & $1 / 16$ & $1 / 16$ \\
\hline
\end{tabular}

ex: exon; WT: wild-type; fs: frame shift; del: deletion. 
Sanger sequencing of selected genes, including FBXW7, NLE, NOTCH1, PHF6, and PTEN, and combined these data with the results of WES. As shown in Table 2, mutations in NOTCH1 signaling pathway genes, including FBXW7, NLE, and NOTCH1, were detected in 4/16 patients. In addition, 8 and 1 of 16 patients exhibited mutations in PHF6 and PTEN, respectively.

\section{Gene expression signature of MEF2D fusion-positive patients}

We have microarray data of B-ALL as indicated in Online Supplementary Figure $S 1,{ }^{11}$ and, in addition, we performed WTS to search for new fusion genes specifically among cases of B-other-ALL. To further assess the func- tional aspects of MEF2D fusions, we performed DNA microarray-based expression profiling. Upon supervised hierarchical clustering analysis using selected gene probe sets (Online Supplementary Table S7 and S8), we observed distinct clustering of $M E F 2 D$ fusion cases, with clear separation from cases with other genetic abnormalities, indicating that $M E F 2 D$ fusion cases have a distinct gene expression signature (Figure 3A). Of note was that this cluster of MEF2D fusion cases was close to that of TCF3$P B X 1$-positive cases. Principal component analysis (PCA) also revealed clear clustering of $M E F 2 D$ fusion cases separate from those cases with other genetic abnormalities, but close to the cluster of TCF3-PBX1-positive cases (Figure 3B).
A

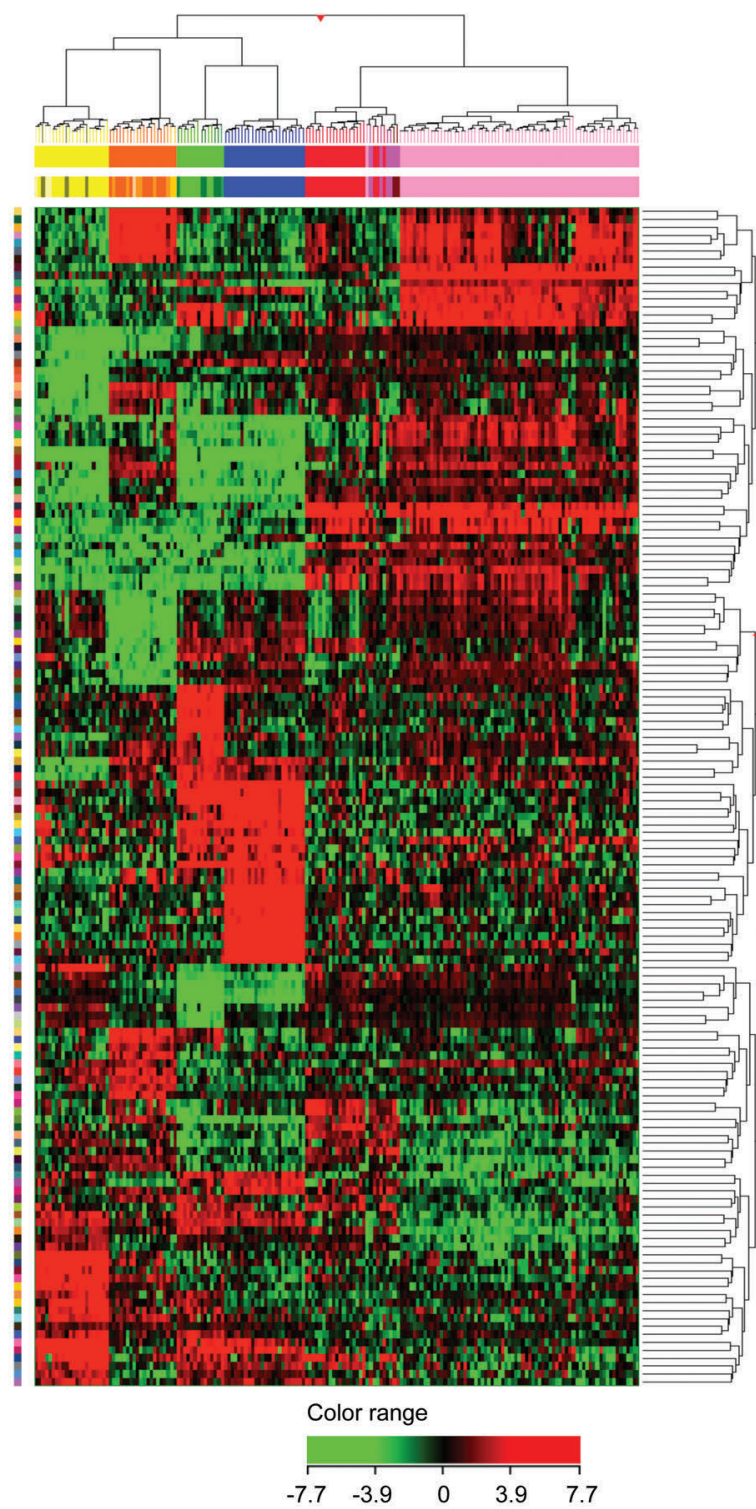

B
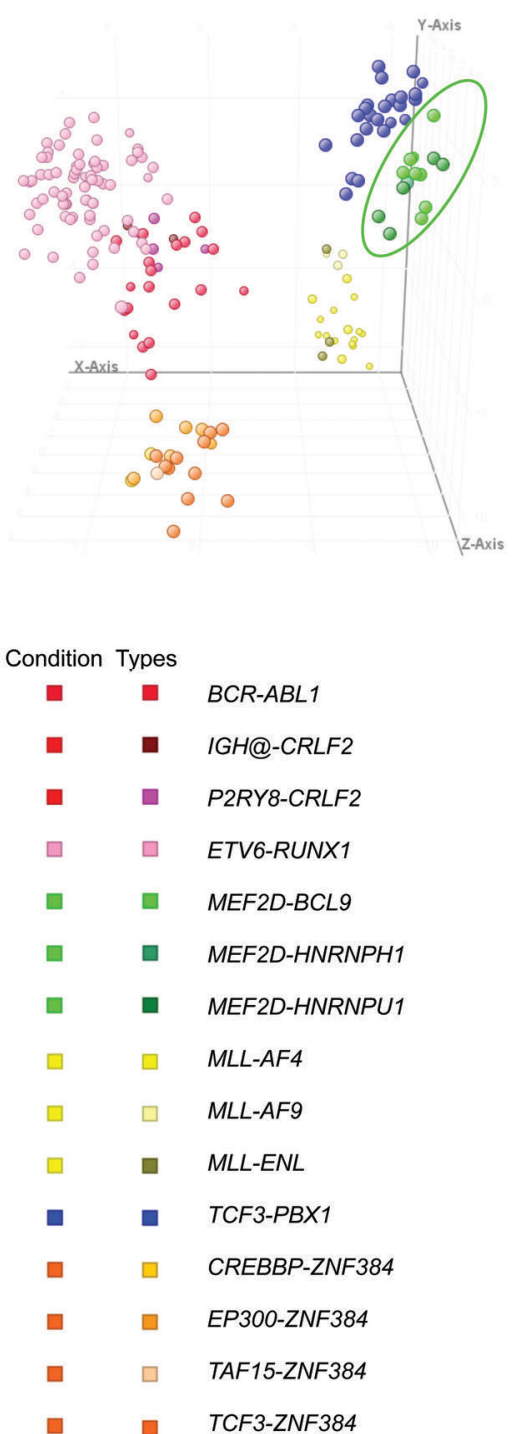

Figure 3. Characteristics of gene expression profile in MEF2D-fusion-positive ALL. (A) Two-way hierarchical clustering and (B) principal component analysis (PCA) were performed on the microarray data, including B-ALLs with MEF2D fusion-positive and other types of genetic abnormalities, using the probe sets of differentially expressed genes between B-ALL with MEF2D fusions and other types of genetic abnormalities selected from filtered microarray probes (presented in Online Supplementary Tables S7 and S8). The results of clustering analysis are displayed using a heat map as a dendrogram. 
As TCF3-PBX1-positive cases also express the cytoplasmic $\mu$ chain-positive pre-B ALL immunophenotype, ${ }^{28,29}$ we subsequently compared the gene expression of $M E F 2 D$ fusion cases with TCF3-PBX1-positive cases as well as Bother-ALL. As shown in Online Supplementary Figure S4, $M E F 2 D$ fusion patients were clearly separate, within a distinct cluster from TCF3-PBX1-positive and B-other-ALL cases, based on unsupervised hierarchical clustering. Upregulated genes common to both $M E F 2 D$ fusion and TCF3-PBX1-positive cases compared to B-other-ALL included: IKZF2, IRF4, TCL6, IGHG, IGHV5-78, IGLL1, VPREB3, and BCL2L11, while RUNX2, IRF9, CRLF2, $C D 34$, and $C C N D 2$ were common down-regulated genes (summarized in Online Supplementary Figure S5). HDAC9 was also identified as a common up-regulated gene in both MEF2D fusion and TCF3-PBX1-positive cases, whereas its expression was significantly higher in $M E F 2 D$ fusion than TCF3-PBX1-positive cases. On the other hand, $M E F 2 D, M M E$ (coding CD10), and RAG1 were down-regulated in MEF2D fusion, but not in TCF3-PBX1-positive cases, thus the low-level expression of CD10 seen in $M E F 2 D$ fusion cases was identified at the gene-expression level. Interestingly, GATA3 was identified as a highly expressed gene in MEF2D fusion cases, yet it was significantly down-regulated in TCF3-PBX1-positive cases.

To explore the gene expression characteristics of $M E F 2 D$ fusion ALL connected with B-cell differentiation, we performed GSEA using 18 curated gene sets of B lymphocytes at various differentiation stages, as well as 7 early hematopoietic stages including stem cells. Firstly, we compared the gene expression signatures of B-ALL cases with different types of genetic abnormalities. Using Bother-ALL as a reference control, we observed that the majority of gene expression signatures found in B lymphocytes at various differentiation stages were enriched in ALL cases with TCF3-PBX1 (Online Supplementary Table $S 10$ and S11). In contrast, only three gene sets were enriched in MEF2D fusion ALL. We further examined the gene expression characteristics of MEF2D fusion ALL by direct comparison with TCF3-PBX1, and observed that most of the signatures of differentiation stage-specific $B$ lymphocytes as well as early hematopoietic populations were enriched in TCF3-PBX1, but not MEF2D fusion ALL (Online Supplementary Table S10).

EFS

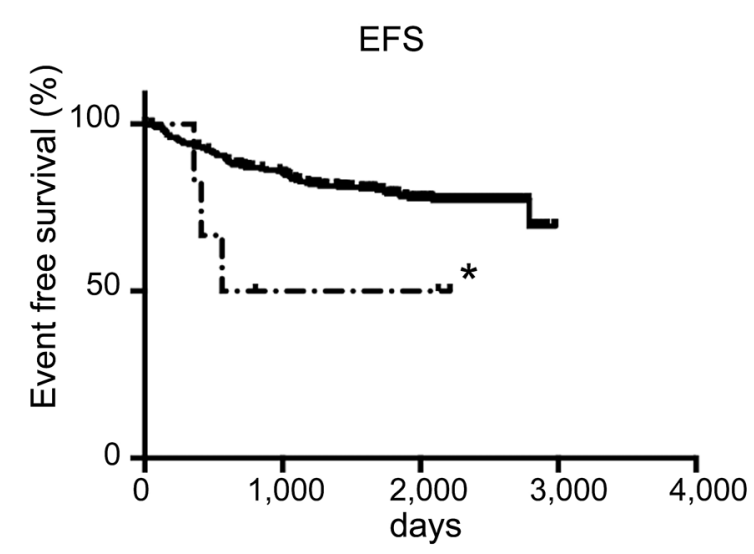

\section{Clinical characteristics and outcomes of MEF2D fusion-positive patients}

The clinical findings and outcome of patients with $M E F 2 D$ fusions are summarized in Table 1. MEF2D fusion B-ALL patients were aged between 3 and 15 years (median: 9 years) at presentation and comprised 6 males and 10 females. Their initial white blood cell (WBC) counts at presentation ranged from 3,400 to 124,100 (median: $27,300 / \mu 1$ ). Analysis of fluids obtained by lumbar puncture revealed no indication of central nervous system (CNS) involvement (data not shown). Among 13 patients, 10 (62.5\%) and 4 $(25.0 \%)$ were classified with an intermediate risk (IR) and high risk (HR), respectively, based on advanced age, elevated WBC counts, or both, and thus standard risk (SR) was assigned to only 2 patients. The response to steroid monotherapy, using the cutoff of $1,000 / \mu \mathrm{L}$ for the blast count in peripheral blood on day 8 was not poor in MEF2D fusion patients; however, among 15 patients, 8 (53.3\%) showed bone marrow or CNS relapse, and all of the relapsed patients died. As shown in Figure 4, both the 5 year EFS and OS rates for MEF2D fusion patients were significantly lower compared with a consecutive series of $\mathrm{B}$ other-ALL patients enrolled onto the L04-16/L06-16 study ( $P=0.0306$ and $P=0.0013$, respectively), indicating that outcomes for $M E F 2 D$ fusion-positive patients were significantly less favorable than those with B-other-ALL.

\section{Discussion}

In this study, we identified MEF2D fusions, including a novel fusion gene, MEF2D-HNRNPH1, at an incidence of approximately $2 \%$ in our B-ALL cohort. It was notable that we identified MEF2D fusions in B-LBL. B-ALL patients with $M E F 2 D$ fusions showed unique clinical and biological characteristics. They exhibited an older age at presentation and elevated WBC counts, thus were mostly classified into the IR or HR groups. Although their response to steroid treatment was not poor, MEF2D fusion patients showed a significantly worse prognosis with more than half of them relapsing and dying within 1 year. It is noteworthy that stem cell transplantation was not effective in any of the five cases where it was administered as a salvage therapy for relapsed patients in our cohort. Therefore, the establish-

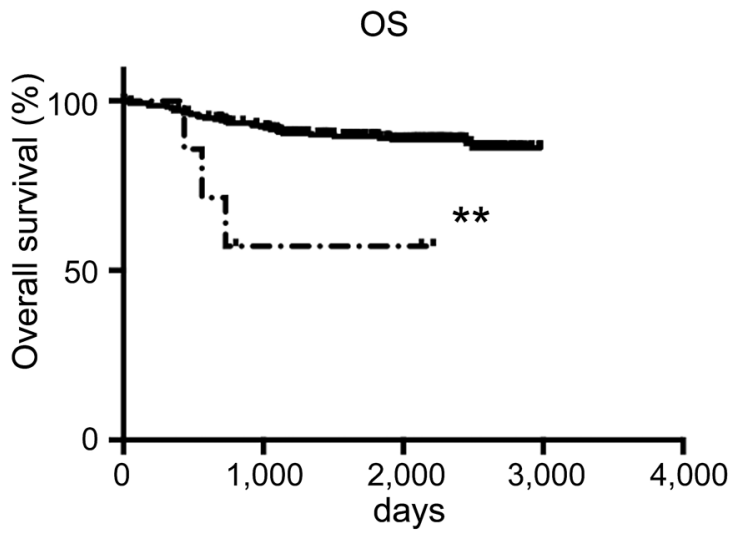

Figure 4. Outcomes of patients with MEF2D fusions. (A) Kaplan-Meier estimates of eventfree survival (EFS) of patients with MEF2D fusions, and B-other, log-rank $P=0.306$ ). (B) Overall survival (OS) for the same as above (log-rank $P=0.0013$ ).

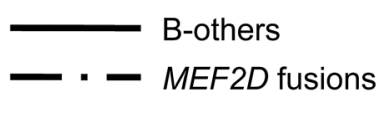

*,P=0.0306 ** $P=0.0013$ 
ment of an early diagnostic method and a new therapeutic strategy are necessary for this type of B-ALL. As ex vivo sensitivity of xenografted leukemic cells harboring MEF2D fusions to HDAC inhibitors has been recently reported, ${ }^{10}$ it may offer a plausible therapeutic option for this type of BALL. In relation to diagnosis, we have already established a qPCR based detection assay for the frequent MEF2D fusions, which may be useful for rapid diagnosis in combination with FISH.

Another interesting observation is that B-ALL cases with MEF2D fusions have a characteristic immunophenotype, most typically presenting as CD5- and cytoplasmic $\mu$ chainpositive. Although it was previously reported that their immunophenotypes were characterized by weak or absent expression of CD10 and high expression of CD38, ${ }^{10}$ we found that CD10 expression was not significantly lower than B-other-ALL, and that CD38 expression level was similar to that of TCF3-PBX1-positive cases. Therefore, B-ALL patients with $M E F 2 D$ fusions could be more effectively diagnosed by combining genetic testing with immunophenotyping.

As shown in Table 1, MEF2D fusion cases were mutually exclusive of the known risk stratifying chromosomal translocations, at least at the cytogenetic level. Among additional genetic alterations in $M E F 2 D$ fusion cases, we identified a significantly higher frequency of CDKN2A/CDKN2B deletions. At initial diagnosis, 7/8 (87.5\%) relapsed patients had CDKN2A/CDKN2B deletions, while they were seen in only $2 / 7(28.6 \%)$ non-relapse patients. Thus, the prognostic impact of CDKN2A/CDKN2B deletions in MEF2D fusion B-ALL needs to be assessed in larger patient cohorts. Interestingly, we also identified that MEF2D fusion patients had mutations in genes known to be recurrent in T-ALL; in particular, there was an unexpectedly high frequency of mutations in PHF6 (8/16). PHF6 encodes a plant homeodomain (PHD) factor with a proposed role in gene expression control. ${ }^{30}$ As PHF6 has been proposed to play a role as a tumor suppressor gene, ${ }^{24}$ it may participate in the pathogenesis of $M E F 2 D$ fusion B-ALL. Thus, further investigation is needed to clarify this role. Additionally, mutations of genes in the RAS pathway (NRAS, KRAS, NF1, and PTPN11) or IKZF1 alterations were not observed in our cases, in conflict with previous reports.

Our data also showed that MEF2D fusion B-ALL has a gene expression signature significantly distinct from other genetic subtypes of B-ALL. Supervised analysis led to separation of $M E F 2 D$ fusion cases into isolated clusters both in PCA and hierarchical clustering, and these clusters were the closest neighbors to TCF3-PBX1-positive cases. This observation is consistent with the knowledge that both types of B-ALL tend to show a pre-B ALL immunophenotype, and high-level expression of pre-BCR components, including IGHG/IGHV 5-78 and IGLL1, were commonly observed in both subtypes. ${ }^{31}$ However, when we examined the gene expression signatures closely, there were significant differences between them.

It has recently been shown that MEF2 family proteins, including MEF2C and MEF2D, play a critical role in early Bcell differentiation. It was demonstrated that B-cell development was blocked at the pre-B-cell stage in Mef2c/d-deficient mice, indicating that they are essential for progression of B-cell precursors (large to small pre-B-cell transition). ${ }^{32}$ It was further shown that, upon activation via pre-B-cell receptor signaling, Mef2c/d induces target genes, including interferon regulatory factor 4 (Irf4; stimulates the expression of
Ikzf1/3) and histone deacetylase 5/9 (Hdac5/9; encoding the protein known to repress Mef2c activity) as we have summarized schematically in Online Supplementary Figure S4. On the other hand, MEF2D fusion B-ALL cases exhibited elevated expression of IRF4 and HDAC9 with down-regulation of $M E F 2 C$, although it has been suggested that deregulated expression of the $\mathrm{N}$ terminus of MEF2D should induce the up-regulation of the target genes of MEF2C/D, whereas subsequent excess of HDAC9 activity represses MEF2C and its downstream gene expression cascade. ${ }^{10}$

Our existing data further revealed that up-regulation of GATA3 is another gene expression characteristic of MEF2D fusion ALL. GATA3 is a critical transcription factor in early T-cell development ${ }^{33-35}$ and its transcriptional repression is essential for early B-cell commitment, ${ }^{36-37}$ while it has also been reported that GATA3 exhibits myeloid-inducing activity in committed B-lymphocytes under the defect of PAX5 function. ${ }^{38-41}$ Furthermore, significantly increased GATA3 mRNA levels were associated with ZNF384 fusion-positive cases and a higher risk of relapse in childhood B-ALL with the Ph-like phenotype. ${ }^{42-43}$ As our data also indicated downregulation of GATA3 expression in TCF3-PBX1-positive cases, ectopic overexpression of GATA3 appears to participate in establishment of a characteristic gene expression signature as well as the biological signature of $M E F 2 D$ fusion ALL distinct from that of other genetic subtypes of B-ALL.

The roles of the MEF2D fusion partners in terms of their biological effects within the fusion molecules of MEF2D fusion ALL are largely unknown. The genes known to be fused to MEF2D in B-ALL have a variety of biological functions. For example, BCL9 is known to be related to WNT/ $\beta$ catenin signaling, whereas the MEF2D-BCL9 fusion retains only the last one or two exons of the BCL9 gene, thus lacking the functional domains required for $W N T / \beta$-catenin signaling, suggesting a role other than deregulation of WNT/ $\beta$ catenin signaling. ${ }^{10}$ HNRNPUL1 encodes a nuclear RNAbinding protein of the heterogeneous nuclear ribonucleoprotein family that may play a role in nucleocytoplasmic RNA transport and DNA repair, ${ }^{18}$ whereas its role as a portion of the fusion molecule in the pathogenesis of B-ALL remains unclear. As HNRNPH1 is a member of the same protein family as HNRNPUL1, both MEF2D-NRNPUL1 and MEF2D-HNRNPH1 likely share the same unknown function.

In conclusion, we have shown that ALL patients harboring $M E F 2 D$ fusion genes possess a characteristic immunophenotype and gene expression signature as well as distinct clinical features, defining them as a distinct genetic subtype among B-other-ALL. Although additional studies are required to elucidate the biological function of the MEF2D fusion protein in leukemogenesis, our data has allowed improved characterization of this new B-ALL subtype.

\section{Acknowledgments}

The authors would like to thank K. Itagaki, H. Yagi, Y. Katayama, A. Tamura, K. Takeda, K. Hayashi, and the staff of the Laboratory for Genotyping Development, Riken Center for Integrative Medical Sciences for their excellent data management and experimental assistance. We thank all members of the Committees of ALL and of Research and Diagnosis of TCCSG.

\section{Funding}

This work was supported in part by a Health and Labour Sciences Research Grant (3rd-term comprehensive 10-year 
strategy for cancer control H22-011), the Grant of the National Center for Child Health and Development (26-20), and the Advanced Research for Medical Products Mining Programme of the National Institute of Biomedical Innovation (NIBIO, 10-41, $-42,-43,-44,-45)$, and Biobank Japan project funded by the Ministry of Education, Culture, Sports, Science and Technology
(MEXT) and the Japan Agency for Medical Research and Development (AMED), and the Practical Research for Innovative Cancer Control from AMED.

These funding sources played no role in the collection, analysis, or interpretation of the results, or in the writing of the manuscript and decision to submit it.

\section{References}

1. Hunger SP, Mullighan CG. Acute lymphoblastic leukemia in children. $\mathrm{N}$ Engl J Med. 2015;373(16):1541-1552.

2. Schwab C, Harrison CJ. Advances in B-cell precursor acute lymphoblastic leukemia genonics. HemaSphere. 2018;2(4):e53

3. Roberts KG, Morin RD, Zhang J, et al. Genetic alterations activating kinase and cytokine receptor signaling in high-risk acute lymphoblastic leukemia. Cancer Cell. 2012;22(2):153-166.

4. Roberts KG, Li Y, Payne-Turner D, et al. Targetable kinase-activating lesions in $\mathrm{Ph}$ like acute lymphoblastic leukemia. N Engl J Med. 2014;371(11):1005-1015.

5. Gocho Y, Kiyokawa N, Ichikawa $H$, et al. A novel recurrent EP300-ZNF384 gene fusion in B-cell precursor acute lymphoblastic leukemia. Leukemia. 2015;29(12):24452448.

6. Liu YF, Wang BY, Zhang WN, et al. Genomic profiling of adult and pediatric B-cell acute lymphoblastic leukemia. EBioMedicine. 2016;8:173-183

7. Yasuda $T$, Tsuzuki $S$, Kawazu M, et al. Recurrent DUX4 fusions in B cell acute lymphoblastic leukemia of adolescents and young adults. Nat Genet. 2016;48(5):569574.

8. Lilljebjörn $\mathrm{H}$, Henningsson R, HyreniusWittsten A, et al. Identification of ETV6RUNX1-like and DUX4-rearranged subtypes in paediatric B-cell precursor acute lymphoblastic leukaemia. Nat Commun. 2016;7:11790

9. Zhang J, McCastlain $\mathrm{K}$, Yoshihara $\mathrm{H}$, et al. Deregulation of DUX4 and ERG in acute lymphoblastic leukemia. Nat Genet. 2016; 48(12):1481-1489.

10. Gu Z, Churchman M, Roberts K, et al Genomic analyses identify recurrent MEF2D fusions in acute lymphoblastic leukaemia. Nat Commun. 2016;7:13331.

11. Hirabayashi S, Ohki K, Nakabayashi K, et al. ZNF384-related fusion genes define a subgroup of childhood B-cell precursor acute lymphoblastic leukemia with a characteristic immunotype. Haematologica. 2017;102 (1):118-129.

12. Prima V, Gore L, Caires A, et al. Cloning and functional characterization of MEF2D/DAZAP1 and DAZAP1/MEF2D fusion proteins created by a variant $\mathrm{t}(1 ; 19)(\mathrm{q} 23 ; \mathrm{p} 13.3)$ in acute lymphoblastic leukemia. Leukemia. 2005;19(5):806-813.

13. Lilljebjörn H, Ågerstam H, Orsmark-Pietras $\mathrm{C}$, et al. RNA-seq identifies clinically relevant fusion genes in leukemia including a novel MEF2D/CSF1R fusion responsive to imatinib. Leukemia. 2014;28(4):977-979.

14. Suzuki K, Okuno Y, Kawashima N, et al MEF2D-BCL9 fusion gene is associated with high-risk acute B-cell precursor lymphoblastic leukemia in adolescents. J Clin Oncol. 2016;34(28):3451-3459.

15. Breitbart RE, Liang CS, Smoot LB, Laheru
DA, Mahdavi V, Nadal-Ginard B. A fourth human MEF2 transcription factor, hMEF2D, is an early marker of the myogenic lineage. Development. 1993; 118(4):1095-1106.

16. Mao Z, Bonni A, Xia F, Nadal-Vicens M, Greenberg M E. (1999) Neuronal activitydependent cell survival mediated by transcription factor MEF2. Science. 1999;286 (5440):785-790.

17. Miska EA, Karlsson C, Langley E, Nielsen SJ, Pines J, Kouzarides T. HDAC4 deacetylase associates with and represses the MEF2 transcription factor. EMBO J. 1999; 18(18): 5099-5107.

18. Honoré $\mathrm{B}$, Rasmussen $\mathrm{HH}$, Vorum $\mathrm{H}$, et al. Heterogeneous nuclear ribonucleoproteins $\mathrm{H}, \mathrm{H}^{\prime}$, and $\mathrm{F}$ are members of a ubiquitously expressed subfamily of related but distinct proteins encoded by genes mapping to different chromosomes. J Biol Chem. 1995; 270(48):28780-28789

19. Takahashi $H$, Kajiwara $R$, Kato $M$, et al. Treatment outcome of children with acute lymphoblastic leukemia: the Tokyo Children's Cancer Study Group (TCCSG) Study L04-16. Int J Hematol. 2018;108:98108.

20. Masuzawa A, Kiyotani C, Osumi T, et al. Poor responses to tyrosine kinase inhibitors in a child with precursor B-cell acute lymphoblastic leukemia with SNX2-ABL1 chimeric transcript. Eur J Haematol. 2014; 92(3):263-267.

21. Kobayashi K, Mitsui K, Ichikawa H, et al. ATF7IP as a novel PDGFRB fusion partner in acute lymphoblastic leukaemia in children. Br J Haematol. 2014;165(6):836-841.

22. McPherson A, Hormozdiari F, Zayed A, et al. deFuse: an algorithm for gene fusion discovery in tumor RNA-Seq data. PLoS Comput Biol. 2011;7(5):e1001138.

23. Weng AP, Ferrando AA, Lee W, et al. Activating mutations of NOTCH1 in human $\mathrm{T}$ cell acute lymphoblastic leukemia. Science. 2004:306(5694):269-271.

24. Van Vlierberghe P, Palomero T, Khiabanian $\mathrm{H}$, et al. PHF6 mutations in T-cell acute lymphoblastic leukemia. Nat Genet. 2010; 42(4):338-342.

25. Wang $\mathrm{Q}$, Qiu $\mathrm{H}$, Jiang $\mathrm{H}$, et al. Mutations of PHF6 are associated with mutations of NOTCH1, JAK1 and rearrangement of SETNUP214 in T-cell acute lymphoblastic leukemia. Haematologica. 2011;96(12): 1808-1814

26. Spinella JF, Cassart P, Richer C, et al. Genomic characterization of pediatric T-cell acute lymphoblastic leukemia reveals novel recurrent driver mutations. Oncotarget. 2016;7(40):65485-65503.

27. Royet J, Bouwmeester T, Cohen SM. Notchless encodes a novel WD40-repeatcontaining protein that modulates Notch signaling activity. EMBO J. 1998; 17(24):7351-7360.

28. Carroll AJ, Crist WM, Parmley RT, Roper M, Cooper MD, Finley WH. Pre-B cell leukemia associated with chromosome translocation 1:19. Blood. 1984;63(3):721-724.
29. Borowitz MJ, Hunger SP, Carroll AJ, et al Predictability of the $t(1 ; 19)(q 23 ; p 13)$ from surface antigen phenotype: implications for screening cases of childhood acute lymphoblastic leukemia for molecular analysis: a Pediatric Oncology Group study. Blood. 1993;82(4):1086-1091

30. Lower KM, Turner G, Kerr BA, et al. Mutations in PHF6 are associated with Börjeson-Forssman-Lehmann syndrome. Nat Genet. 2002;32(4):661-665.

31. Chen D, Zheng J, Gerasimcik N, et al. The expression pattern of the Pre-B cell receptor components correlates with cellular stage and clinical outcome in acute lymphoblastic leukemia. PLoS One. 2016; 11(9):e0162638.

32. Herglotz J, Unrau L, Hauschildt F, et al Essential control of early B-cell development by Mef2 transcription factors. Blood. 2016;127(5):572-581.

33. Landry DB, Engel JD, Sen R. Functional GATA 3 binding sites within murine CD8 alpha upstream regulatory sequences. J Exp Med. 1993;178(3):941-949.

34. Ting CN, Olson MC, Barton KP, Leiden JM. Transcription factor GATA-3 is required for development of the T-cell lineage. Nature. 1996;384(6608):474-478.

35. Gao J, Chen YH, Peterson LC. GATA family transcriptional factors: emerging suspects in hematologic disorders. Exp Hematol Oncol. 2015;4:28.

36. Banerjee A, Northrup D, Boukarabila H, Jacobsen SE, Allman D. Transcriptional repression of Gata3 is essential for early B cell commitment. Immunity. 2013; 38(5):930-42.

37. Somasundaram R, Prasad MA, Ungerbäck J, Sigvardsson M. Transcription factor networks in B-cell differentiation link development to acute lymphoid leukemia. Blood. 2015;126(2):144-152.

38. Chen D, Zhang G. Enforced expression of the GATA-3 transcription factor affects cell fate decisions in hematopoiesis. Exp Hematol. 2001;29(8):971-980.

39. $\mathrm{Ku} \mathrm{CJ}$, Hosoya T, Maillard I, Engel JD. GATA 3 regulates hematopoietic stem cell maintenance and cell cycle entry. Blood. 2012;119(10):2242-2251.

40. Frelin $C$, Herrington $R$, Janmohamed $S$, et al. GATA 3 regulates the self renewal of long term hematopoietic stem cells. Nat Immunol. 2013;14(10):1037-1044

41. Heavey B, Charalambous C, Cobaleda C, Busslinger M. Myeloid lineage switch of Pax5 mutant but not wild-type B cell progenitors by $\mathrm{C} / \mathrm{EBPalph}$ and GATA factors. EMBO J. 2003;22(15):3887-3897.

42. Perez-Andreu V, Roberts KG, Harvey RC, et al. Inherited GATA3 variants are associated with Ph-like childhood acute lymphoblastic leukemia and risk of relapse. Nat Genet. 2013;45(12):1494-1498.

43. Perez-Andreu V, Roberts KG, Xu H, et al. A genome-wide association study of susceptibility to acute lymphoblastic leukemia in adolescents and young adults. Blood. 2015; 125(4):680-686 